\title{
Study on viscosity induced contrast in ultrasound color flow imaging of carotid atherosclerosis
}

\author{
A. B. M. Aowlad Hossain 1 , Dabasish Kumar Saha', Zubair Ahmed Ratan ${ }^{3}$ \\ ${ }^{1}$ Department of Electronics and Communication Engineering, Khulna University of Engineering and Technology, \\ Bangladesh \\ ${ }^{2,3}$ Deptartment of Biomedical Engineering, Khulna University of Engineering and Technology, Bangladesh
}

\begin{tabular}{l} 
Article Info \\
\hline Article history: \\
Received Aug 13, 2020 \\
Revised Jan 29, 2021 \\
Accepted Mar 5, 2021 \\
\hline
\end{tabular}

\section{Keywords:}

Carotid atherosclerosis Computational fluid dynamics Ultrasound color flow imaging Viscosity induced contrast

\begin{abstract}
Efficient imaging of blood flow disturbances resulted from carotid atherosclerosis plays a vital role clinically to predict brain stroke risk. Carotid atherosclerosis and its development is closely linked with raised blood viscosity. Therefore, study of viscosity changing hemodynamic effect has importance and it might be useful for improved examination of carotid atherosclerosis incorporating the viscosity induced contrast in conventional ultrasound imaging. This work considered the design of realistic models of atherosclerotic carotid artery of different stages and solved to compute the hemodisturbances using computational fluid dynamics (CFD) by finite element method (FEM) to investigate viscosity changes effect. Ultrasound color flow image of velocities of blood have been constructed using phase shift information estimated with autocorrelation of Hilbert transformed simulated backscattered radiofrequency (RF) signals from moving blood particles. The simulated ultrasound images have been compared with CFD simulation images and identified a good match between them. The atherosclerosis stages of the models have been investigated from the estimated velocity data. It has been observed that the blood velocities increase noticeably in carotid atherosclerotic growths and velocity distribution changes with viscosity variations. It is also found importantly that the viscosity induced contrast associated to atherosclerosis is detectable in ultrasound color flow imaging. The findings of this work might be useful for better investigation of carotid atherosclerosis as well as prediction of its progression to reduce the stroke risk.
\end{abstract}

This is an open access article under the CC BY-SA license.

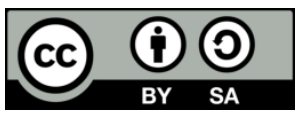

Corresponding Author:

A. B. M. Aowlad Hossain

Department of Electronics and Communication Engineering

Khulna University of Engineering and Technology

Khulna-9203, Bangladesh

Email: aowlad0403@ece.kuet.ac.bd

\section{INTRODUCTION}

Stroke is one of the major cause of mortality in the world and about $70 \%$ of stroke occur in the lower-middle-income countries and has been the main cause of disability [1]. Atherosclerosis is an inflammatory situation in which plaque accumulates in arterial walls that can cause narrowing the artery as well as harden the artery wall that results in loss of elasticity in the arteries. This phenomenon restricts the influx of oxygen-rich blood to our organs and carotid atherosclerosis is a cause of stroke worldwide [2], [3].

Carotid arteries deliver oxygen-rich blood to the superior portion of the human body. It is divided into two parts: Internal carotid arteries (ICA) and external carotid arteries (ECA). Internal carotid arteries are 
responsible for the supply of oxygenated blood to our brain and blood to our neck, face, and scalp is by the external carotid arteries. If atherosclerosis takes place in these vital arteries, the oxygenated blood flows into our brain hamper and may lead to stroke. Recent studies revealed that blood viscosity is a significant factor, which is related to atherosclerosis and the viscosities found to be elevated in patients with carotid atherosclerosis [4], [5]. Viscosity measures the thickness and stickiness of blood. Elevated blood viscosity is significant biological parameter which has a close relation with the other major risk factors low high density lipoproteins (HDL), high low density lipoproteins (LDL) cholesterol, obesity, diabetes, and metabolic syndrome [6]. Blood viscosity also has an impact on blood pressure and increased blood pressure is responsible for atherosclerosis growth and becomes more risky after the age of 45 [7], [8]. As the progression of the disease, the visco-elastic properties of the artery wall also changes. With the help of the information of carotid hemodynamics due to atherosclerosis, we can examine the relatedness between the level of stenosis of the artery that creates complex hemodynamics and syndromes and, ultimately, the risk of stroke. Hence appropriate understanding and proficient imaging of the blood flow behaviour can help in identifying carotid abnormalities in the initial phase and assessing the progression of stroke risk.

Diagnostic ultrasound imaging has gained widespread applications for screening cardiovascular disease through velocity estimation because of its low cost, fast scan and portable facilities [9]-[11]. But atherosclerosis growth related viscosity induced hemodisturbance contrast is not considered yet in common ultrasound scanning. In this study, we have focused on carotid arteries comparing to other cardiovascular parts because of its susceptibility to atherosclerosis and due to the easy access of this location with the ultrasound probe. Direct investigation on viscosity change effects of carotid atherosclerosis with experimental setup requires physical subjects with known viscosities during normal and abnormal stages which is tedious and error-suspecting process. Simulation and computational study with realistic considerations is a possible and comfy alternative, which are also common practice in such cases [12]-[16].

This study represents a framework that couples with computational fluid dynamics and ultrasound color flow imaging simulations. This work is an extension of our previous preliminary study [17] with more rigorous analysis and more number of realistic models of normal, moderate-abnormal, and severe abnormal carotid artery categories. Ultrasound RF signals have been simulated considering realistic flow patterns in complex vessel geometries. To the best of our knowledge, there are very few studies on ultrasound-based tracking of viscosity changed hemodynamics effects related to carotid atherosclerosis. With this viewpoint, we have focused to observe the impact of viscosity change on blood flow in atherosclerotic carotid artery using CFD concept and examine the effectiveness of viscosity induced contrast to diagnose carotid atherosclerosis and its evolution through ultrasound color flow images. As there is a relationship between elevated blood viscosity and atherosclerosis and its progression, based on the information of the hemodynamics in atherosclerotic artery with various blood viscosities can elucidate the connection between stenosis at artery, related symptoms and severity stages.

The remaining part of this paper is organized as follows. In the methods section, realistic normal and abnormal carotid artery blood flow models are developed and solved using CFD concept. Consequently, simulation techniques of ultrasound color flow image formation from CFD data are described. Then a rigorous analysis on the simulation results are presented in the result analysis and discussion section which is then concluded mentioning the important findings, usefulness and future perspectives.

\section{METHODS}

The whole simulation study is divided into two parts: CFD simulation is as the first part and the ultrasound simulation based on CFD data is the second part. The hemodynamics involved with atherosclerosis can be studied through the coordination of CFD and FEM. Then, we will import geometry information of blood flow model, displacement and velocity data that are generated through numerical computation and will apply effective methods to simulate ultrasound color flow images. In this work, we solve the blood flow geometrical model with the help of finite element method. We applied CFD to numerically solve the differential equations involved with fluid flows. The blood particles location for the different instant of time is essential for velocity calculation. The velocity can be calculated through the movement data of blood particle scatterers from ultrasound RF signals. Finally, the ultrasound-based flow images will be compared with CFD references and viscosity change effects investigated for different carotid artery models. Figure 1 shows the flowchart of the overall simulation methodology of this study.

\subsection{CFD simulations}

The blood is considered as incompressible, laminar and non-Newtonian fluid and Navier-Stokes are used for governing the blood flow model in the carotid. Navier-Stokes equations explicate the fluid flow mechanics governing the dynamical balance between two internal forces due to fluid pressure and viscosity 
with the forces applied externally [18], [19]. Assuming the isothermal conditions the dynamic blood flow is governed by the momentum and mass conservation equation which is represented as:

$$
\begin{aligned}
& \rho(d u / d t+u . \nabla u)=\nabla \sigma+f \\
& \nabla . u=0
\end{aligned}
$$

Where $u$ denotes the velocity, $\sigma$ denotes the stress, density of blood is represented by $\rho$ and $f$ is the volume force per unit mass of blood.

In this study, a fixed density considering the realistic density of blood and variable viscosity related to carotid atherosclerosis has been chosen for the analysis. Dirichlet boundary conditions are assumed for the entrance flow with a time-dependent parabolic velocity distribution. The finite element solver has solved the time-dependent incompressible Navier-Stokes equations to compute the resultant velocities considering the blood properties and boundary conditions. CFD is very popular for vascular flow analysis like the study of flow dynamics of stenosed carotid artery [20]. CFD technique has been used in this study to solve the differential equations of fluid dynamics numerically for computing blood flow data. We have used the widely accepted finite element solver ANSYS software (ANSYS Inc., Canonsburg, USA) to solve blood flow model for getting the blood flow data.

We have developed 2D models of realistic atherosclerotic stenosed carotid artery since our main focus is on the viscosity change effect rather than complex 3D structure and computations. Three models with different cases have been considered relating to normal, mild and severe carotid atherosclerosis cases with no blockage, less than $50 \%$ blockage, and greater than $50 \%$ blockage respectively. Computational models of carotid arteries with main common carotid artery (CCA), internal branch ICA and external branch ECA is modelled through ANSYS. The length of the CCA, ICA and ECA are chosen as $10 \mathrm{~mm}, 5 \mathrm{~mm}$ and $5 \mathrm{~mm}$ respectively. The diameter of the CCA, ICA and ECA are $4 \mathrm{~mm}, 3 \mathrm{~mm}$ and $2 \mathrm{~mm}$ respectively. The stenosis is added in the junction of ICA and ECA, which is known as carotid bifurcation region. The entry level of CCA is denoted as inlet and the end boundaries of ICA and ECA are denoted as outlet boundary. Figure 2 shows three different carotid artery models with CCA, ICA, and ECA along with their real scenarios.

In this study, blood density is chosen as $1060 \mathrm{~kg} / \mathrm{m}^{3}$. Then three different viscosities of $3.5 \mathrm{mPa}-\mathrm{s}$, $7 \mathrm{mPa}-\mathrm{s}$ and $10 \mathrm{mPa}-\mathrm{s}$ have been chosen to analyze the change effects. The incompressibility condition is employed being blood is very well approximated as a perfectly incompressible material. Reynolds numbers for three different blood viscosities with fixed blood density are shown in Table 1.

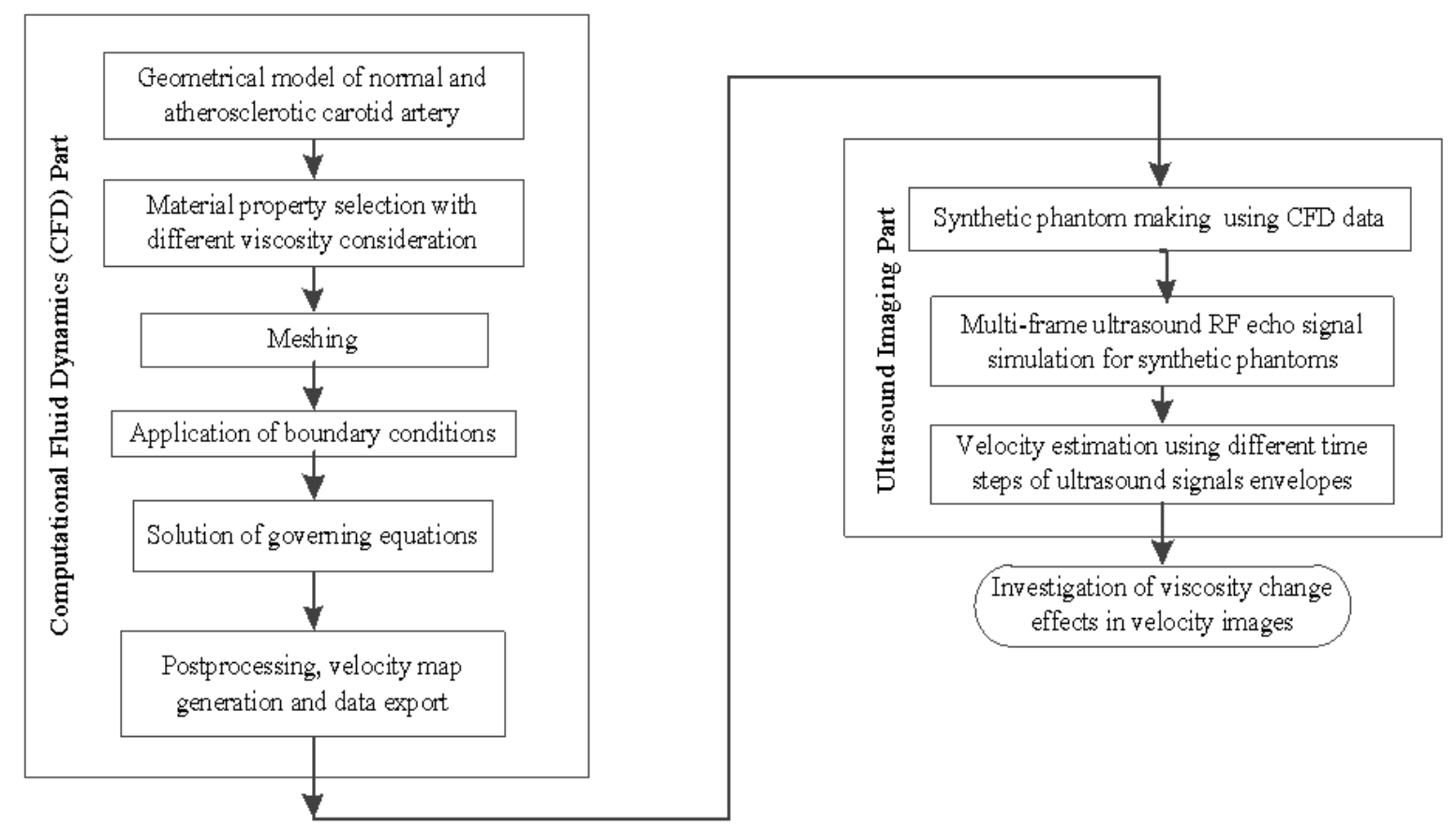

Figure 1. Flowchart of methodology 
Table 1. Blood viscosities and Reynolds numbers for the models

\begin{tabular}{ccc}
\hline Cases & Blood viscosity (mPa-s) & Reynolds number at CCA inlet \\
\hline Normal Carotid & 3.5 & 605 \\
Abnormal Carotid-1 & 3.5 & 605 \\
& 3.5 & 605 \\
Abnormal Carotid-2 & 7.0 & 302 \\
& 10 & 212 \\
\hline
\end{tabular}
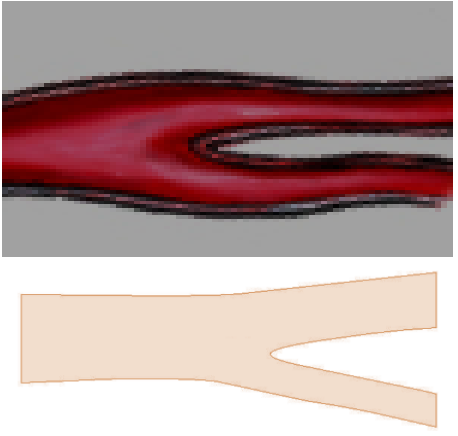

(a)
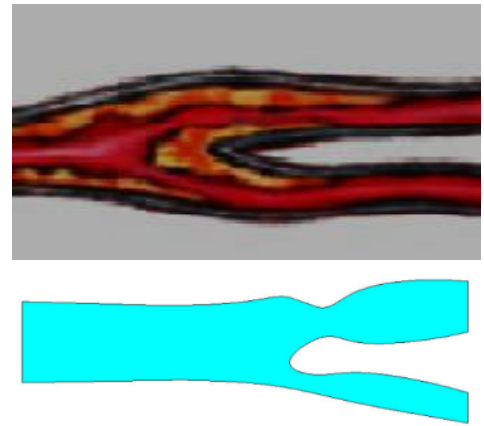

(b)

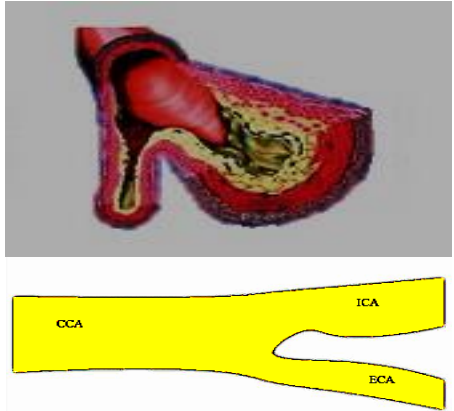

(c)

Figure 2. Carotid artery models for different cases (bottom row) with corresponding realistic scenarios (top row); (a) Normal carotid artery, (b) abnormal carotid artery-1, (c) abnormal carotid artery-2

Meshing criteria has been chosen with a largest gap of $0.040 \mathrm{~mm}$ in the laminar flow sections of the CCA inlet and ECA outlet, and a largest gap of $0.020 \mathrm{~mm}$ in the complex area surrounding the bifurcation, carotid bulb and downward areas of the ICA subdivision. The designed carotid artery geometry models have been meshed into almost 17500 elements. The mesh diagram of carotid artery models for normal and atherosclerosis cases are shown in Figure 3.

Boundary conditions have been applied to the inlet surface, the outlet surfaces and the luminal wall surface. Here, we consider the artery wall as rigid and the velocity profile as parabolic with a maximum velocity of $0.5 \mathrm{~m} / \mathrm{s}$ as an inlet boundary condition. The outlet boundary is defined as a pressure outlet with no initial pressure. Since our main focus to analyze the disturbance occurred due to the atherosclerotic obstacles and to examine those regions in constructed ultrasound velocity images, the blood and artery wall interaction has not been considered. The blood flow velocities are then computed solving the carotid artery blood flow models using ANSYS at different locations in the common carotid and internal and external branches.

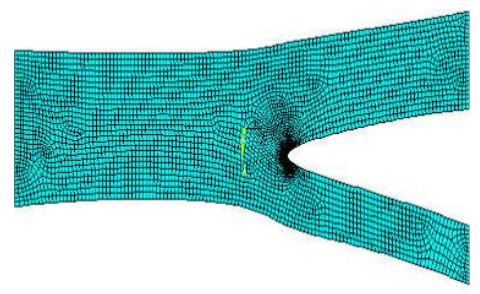

(a)

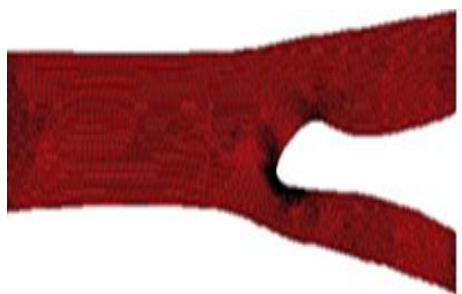

(b)

Figure 3. Mesh diagrams of different carotid artery models (enlarged view); (a) Normal artery, (b) abnormal artery-2

\subsection{Simulation of ultrasound color flow images}

For ultrasound simulations, we have used the well-accepted simulation software Field II [21]-[23]. Field II program has been chosen here because of its perfect modelling of arbitrary probes, beamforming, wave propagation and interactions, and its capability for simulating real like ultrasound images through generating RF signals using the CFD data. This part of the simulation was done using MATLAB (The MathWorks Inc., Massachusetts, USA) and Field II can simulate different kinds of ultrasound transducers, arrays, raw radiofrequency (RF) lines and the images in this platform. In Field II, the red blood cells are 
considered as point scatterers and the blood is modelled as a collection of these scatterers distributed in random manner. The CFD simulation data provided these scattering points to make the synthetic phantoms for generating an ultrasound RF signal and blood flow image. We have to know the position of the scatterer for every time steps for the velocity measurement. The estimation of velocity can be done using the movement data of the scatterers calculated from ultrasound RF signals.

In this study, we modelled a linear ultrasound probe with $5 \mathrm{MHz}$ centre frequency. We used 128 probe elements among them 64 active elements are excited with dynamic focusing strategy to scan the whole synthetic phantom area and simulated $256 \mathrm{RF}$ lines for imaging. Apodization is used using the Hanning window for both transmittinig and receiving aperture. To capture the blood positions, 10 time-steps have been considered for generating RF signals frames using CFD data at that time.

Nowadays, color flow imaging (CFI) is a popular technique to examine the flow dynamics of blood in different clinical situations. The ultrasound CFI show the estimated axial velocity components along the ultrasound beam. The rate of change of phase in RF signal envelope of consequent time instants can be inferred as a time or frequency shifting of the signals and the blood velocity can be calculated from this phase data [24]-[28]. The time shift can be estimated by applying autocorrelation or crosscorrelation of RF echo signals. In this study, we have used an auto-correlation based algorithm [27] to estimate blood velocity and hence to reconstruct CFI. The velocity can be measured from the phase shifting information by taking multiple RF envelopes at different time steps along a single line keeping the transducer fixed and then compute the average phase shifting at each range bin along the line. The change in phase of Hilbert transformed demodulated complex RF signal $x(t)+j y(t)$ for each range bin $t$ can be computed as:

$$
\frac{d \phi(t)}{d t}=\tan ^{-1}\left(\frac{\sum_{e=0}^{E-2}\left[y_{e}(t) x_{e+1}(t)-x_{e}(t) y_{e+1}(t)\right]}{\sum_{e=0}^{E-2}\left[x_{e}(t) x_{e+1}(t)+y_{e}(t) y_{e+1}(t)\right]}\right)
$$

Where $E$ is the ensemble size, varying from 4 to 16 and $e+1$ means the next event of RF acquisition after $e$.

The blood velocity is proportional to the derivative of phase angle which is expressed as:

$$
v_{z}=-\frac{d \phi(t)}{d t} \cdot \frac{c}{4 \pi f_{0}}
$$

If the time between the recorded lines is $T_{p r f}$., then the velocity is expressed as:

$$
v_{z}=-\frac{c}{4 \pi f_{0} T_{p r f}} \tan ^{-1}\left(\frac{\sum_{e=0}^{E-2}\left[y_{e}(t) x_{e+1}(t)-x_{e}(t) y_{e+1}(t)\right]}{\sum_{e=0}^{E-2}\left[x_{e}(t) x_{e+1}(t)+y_{e}(t) y_{e+1}(t)\right]}\right)
$$

The $T_{p r f}$ has been chosen as $1 \mathrm{~ms}$ and 10 pulses are considered. Finally, the ten velocity image frames are averaged to obtain the color flow image. A low pass Gaussian filter with a $5 \times 5$ kernel and unity standard deviation is used to denoise the velocity image. The kernel of the Gaussian filter is calculated as [29]:

$$
G(x, y)=\frac{1}{2 \pi 2 \sigma^{2}} e^{\frac{-\left(x^{2}+y^{2}\right)}{2 \sigma^{2}}}
$$

Where $x, y$ is the pixel positions of the kernel. The filter kernel is gone through all over the constructed raw image of blood velocity and convolved to increase the quality of the image.

\section{RESULTS ANALYSIS AND DISCUSSION}

The FEM model for normal and abnormal atherosclerosis cases has been solved using ANSYS to verify the matching of the ultrasound and CFD results as well as to investigate the viscosity change effects. The obtained CFD based flow images of different models under different viscosity conditions are used as reference images and compare with the simulated ultrasound based flow images to investigate its effectiveness on viscosity variation tracking. Finally visual inspection and quantitative assessment has been done and discussed accordingly. 
Figure 4 shows the normal and atherosclerotic carotid artery bifurcation models with CCA, ICA and ECA and its axial-directional computed velocity map considering a fixed density of $1060 \mathrm{~kg} / \mathrm{m}^{3}$ and viscosity of $3.5 \mathrm{mPa}$-s. The velocity distribution contour in the inlet was found as parabolic. The velocities in the normal carotid models are almost uniformly distributed. But the abnormal carotid models show the hemodisturbance and blood velocity increased significantly in the plaque regions. Swirls have also been noticeable in the bifurcation. Most importantly, higher flow with velocity has been fairly noticed in the tapered regions created by plaque which are usually found real cases also.

As blood viscosity is related with atherosclerosis development, data related to viscosity can improve the quality of ultrasound carotid atherosclerosis flow image. Therefore, CFD images with varying viscosities have been constructed to examine the viscosity variation haemodynamic effect as well as the ability of ultrasound scanning to trace this viscosity variation. For analysis and to understand the growth of carotid atherosclerosis properly, we used three different viscosities of $3.5 \mathrm{mPa}-\mathrm{s}, 7 \mathrm{mPa}-\mathrm{s}$ and $10 \mathrm{mPa}$-s with a fixed density of $1060 \mathrm{~kg} / \mathrm{m}^{3}$ in abnormal carotid-2.

The simulation images from CFD data considering three different defined viscosities in abnormal carotid-2 are shown in Figure 5. It is observed that although inlet velocity was $0.5 \mathrm{~m} / \mathrm{s}$, the peak velocity at atherosclerosis region reaches to about $2.5 \mathrm{~m} / \mathrm{s}$ and haemodisturbance occured due to plaque. From Figure 5, it also observed that with the increases of viscosity the magnitude of distributed velocities are reduced.

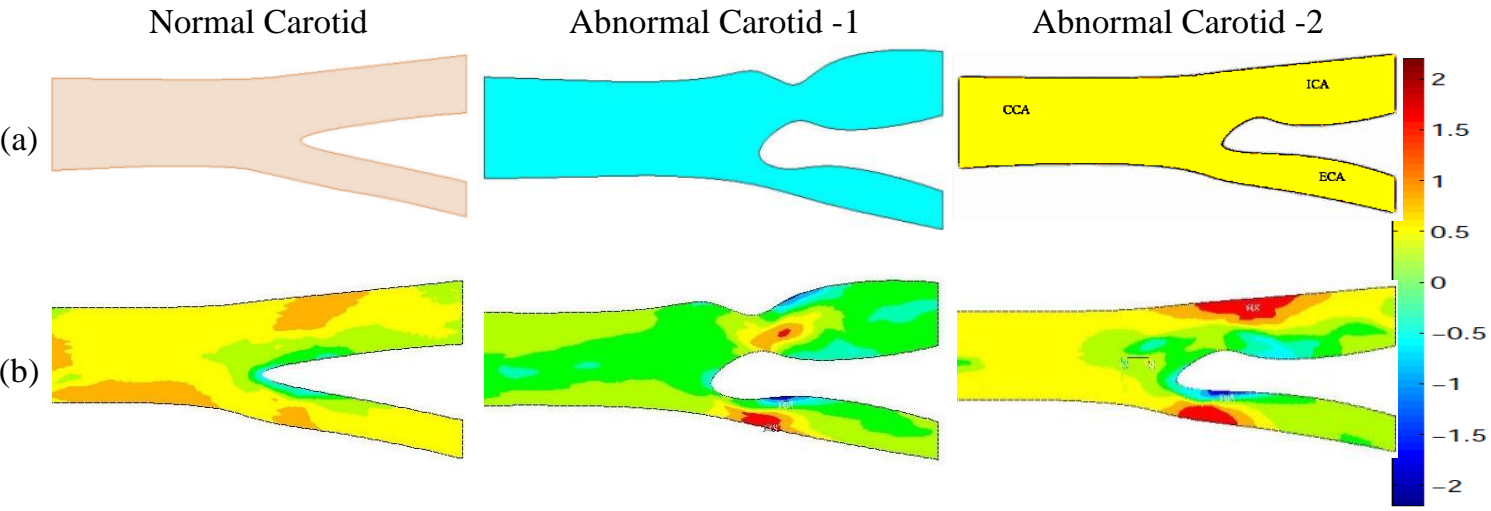

Figure 4. This figure are, (a) Different carotid artery models, (b) CFD computed velocity (m/s) images

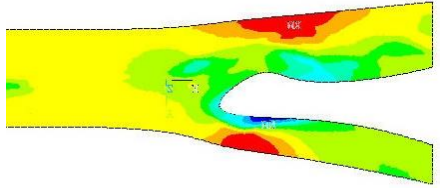

(a)

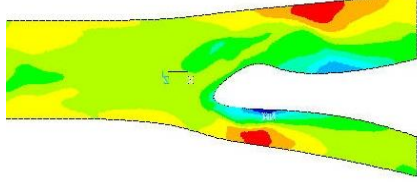

(b)

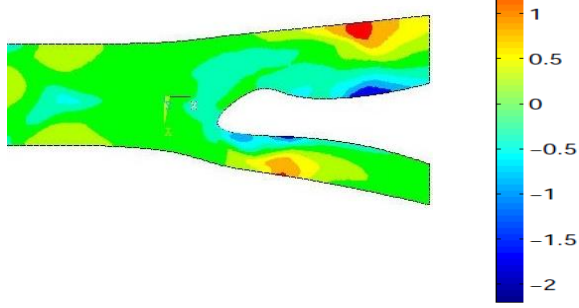

(c)

Figure 5. CFD simulated velocity (m/s) images of abnormal carotid-2 model for viscosities of (a) $3.5 \mathrm{mPa}-\mathrm{s}$, (b) $7 \mathrm{mPa}-\mathrm{s}$ and (c) $10 \mathrm{mPa}-\mathrm{s}$

The color images of the estimated CFD velocities are used as references for the comparison and justification of the performance of the constructed ultrasound color flow imaging to achieve the objectives of this study. For ultrasound images, the velocity images are computed by using the autocorrelation based phase shift estimation algorithm. For comparative analysis, the ultrasound flow images and the reference CFD images for different models are shown in Figure 6. In the ultrasound color flow images, a parabolic distribution of velocity was observed which leads a good match with CFD simulations despite a slight reduction of the velocities. For better analysis, the vector plot of CFD images with an enlarged view is shown 
in Figure 7. It was observed that the flow direction deviated even in the opposite directions which indicate the negative velocity index.

Model

(a)

Normal Carotid

(b) Abnormal Carotid-1
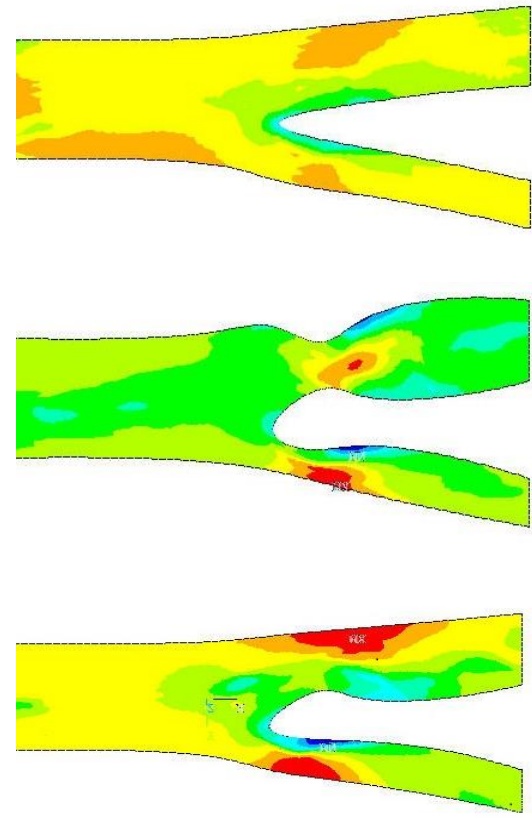

Ultrasound simulated flow image
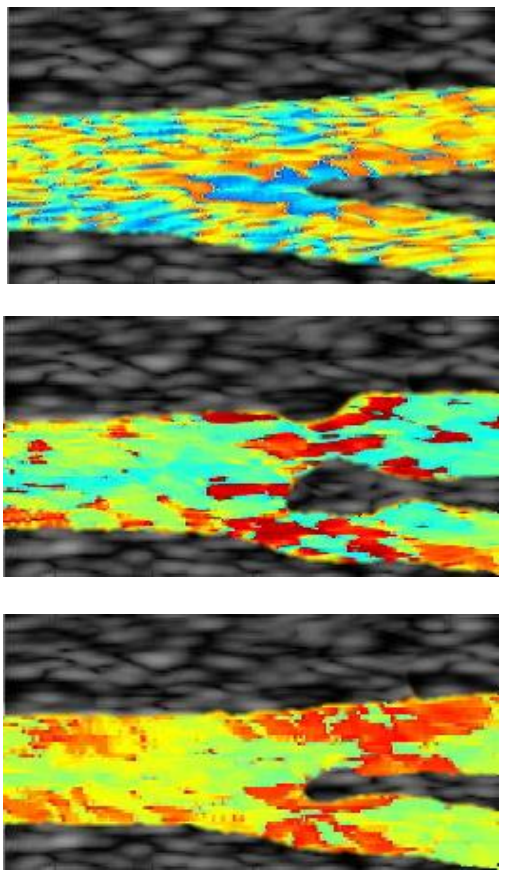

Figure 6. Comparison of CFD computed and ultrasound simulated color flow velocity (m/s) images for different models

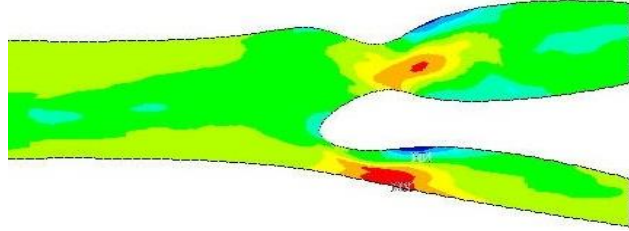

(a)

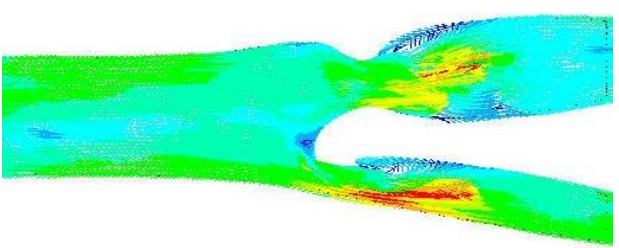

(b)

Figure 7. This figure are; (a) Contour plot and (b) Vector plot of velocity (m/s) distribution of abnormal model-1 with enlarged view

To examine the ability of ultrasound technique to distinguish viscosity changes, simulated ultrasound colour flow images with varying viscosities has been compared with the reference CFD images as shown in Figure 8. In the topmost CFD simulated image with normally considered viscosity is $3.5 \mathrm{mPa}-\mathrm{s}$, it was found that the velocity of blood is normal range in CCA region. But elevated velocity slopes were observed in radial direction to the at the artery wall of ICA and ECA close to the apex part owing to plaque in bifurcated area which constricted the blood vessel. The obtained velocity map from ultrasound CFI illustrate good match with the CFD images with slight variations arises due to speckle noises available in ultrasound scanning.

Blood viscosity was set to an increased value as $7 \mathrm{mPa}$-s for the middle frame images and it was found that the resistance rises due to viscosity elevation. In consequence, fall in velocity in CCA branch and complex haemedisturbance in bifurcation area were found which leads to further development of atherosclerosis. The drops of velocity were found also in ICA and ECA close to the apex-induced place. Supply of blood might be reduced to the brain which may lead probability of stroke. The constructed ultrasound CFI shows a similar trend of CFD reference despite little variations. 
The blood viscosity was then set to more value in simulation study as $10 \mathrm{mPa}$-s in the bottom row of Figure 8 and it was observed that the resistance against flow is extremely raised for additional increasing of viscosity. In consequence, blood velocity drops further in CCA part and a noticeable turbulence flow is created in the bifurcation that further develops atherosclerosis as well as constricted the artery. The velocity of blood drops further in ICA part and ECA close to the tip of partition and blood flow become noticeably lower which leads to the threat of stroke. This ultrasound CFI also showed good match with the CFD image.

The blood velocities in the atherosclerotic regions have been observed higher in both CFD computed and ultrasound based simulated velocity images which are also satisfying the practical happenings. Nevertheless, noises have been observed in the ultrasound based flow images despite the Gaussian filtering. The noises might be induced from speckles as well as truncation process like limited meshing and nodes concern. The magnitude of velocity vector-sum considering all components in the atherosclerotic regions found about 2 to 5 times speedy than that in inlet.

Abnormal model-2

(a) Viscosity: $3.5 \mathrm{mPa}-\mathrm{s}$

(b)

Viscosity: $7 \mathrm{mPa}-\mathrm{s}$

(c)

\section{Viscosity: $10 \mathrm{mPa}-\mathrm{s}$}

CFD simulated flow image
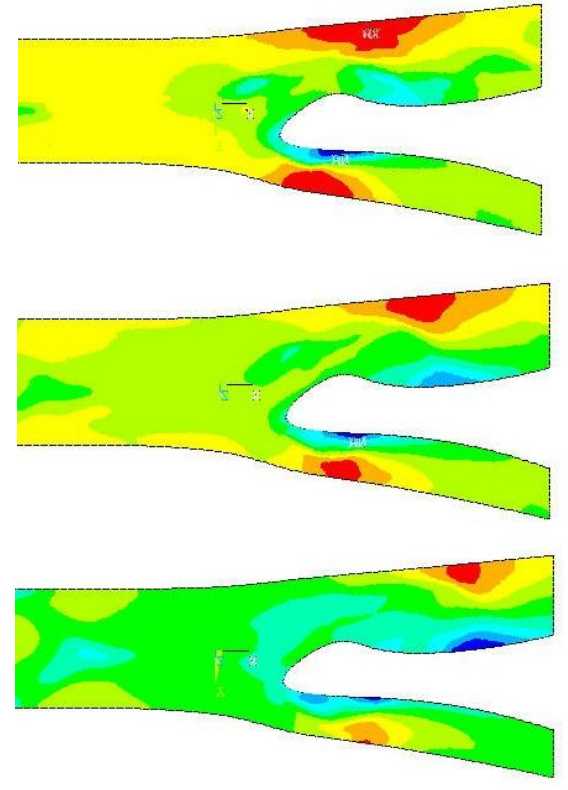

Ultrasound simulated flow image
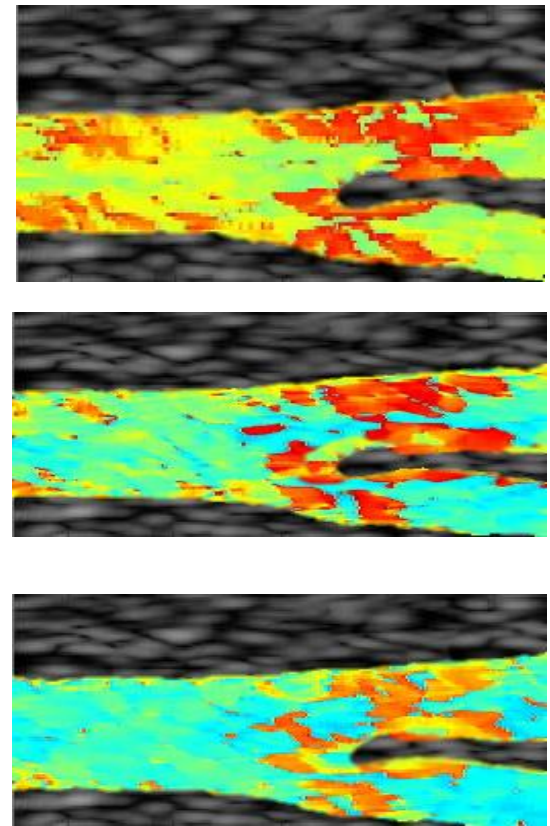

Figure 8. Comparison of CFD and ultrasound images considering viscosity variation in abnormal carotid model-2; (a) Viscosity: $3.5 \mathrm{mPa}-\mathrm{s}$, (b) viscosity: $7 \mathrm{mPa}-\mathrm{s}$, (c) viscosity: $10 \mathrm{mPa}-\mathrm{s}$

From the aforementioned results analysis we can say that as viscosity changes are associated with the growth of carotid atherosclerosis, tracking these changes and incorporation of this additional information can be useful to improve the ultrasound flow imaging quality. The variation of hemodynamical disturbances occurs in bifurcations plaques due to the variation of blood viscosities and sensibly differ from each other. It is found that the velocity images are changed with the changes of viscosity for both cases CFD images and ultrasound color flow images and more complex pattern is found with elevated viscosity. Though ultrasound technique is widely used for carotid atherosclerosis diagnosis, atherosclerosis related viscosity changes effects are not considered in conventional ultrasound imaging. Therefore, effective estimation of this viscosity variation induced velocity changes data using ultrasound technique might provide useful contrast. This additional contrast can improve the ultrasound color flow imaging support accompanied by its innate faster scanning ability for better diagnossis of carotid atherosclerosis.

Furthermore, the atherosclerosis stages of the models have been determined from the estimated velocity data of CFI following standard grading criteria of carotid stenosis [30]. Table 2 shows the peak velocity in the plaque regions of abnormal carotid models with their estimated atherosclerosis stages. Depending on the peak velocity at ICA region of interests and the ICA: CCA velocity, the abnormal carotid model-1 shows the stenosis in severe level according to the referred criteria. The abnormal carotid model-2 with increasing viscosities are estimated as moderate stages with reducing peak velocities, but as the 
viscosity increases the ICA: CCA velocity ratios are increasing towards severe stage which reflects the close relation of viscosity elevation with atherosclerosis progression.

Table 2. Variation of blood velocity for different stenosis

\begin{tabular}{cccc}
\hline Models & ICA peak velocity $(\mathrm{cm} / \mathrm{sec})$ & ICA/CCA velocity ratio & Degree of Stenosis \\
\hline Normal Carotid & $116(<125)$ & $1.2<2.0$ & Normal \\
Abnormal Carotid-1 & $333(>230)$ & $4.7(>4.0)$ & Severe \\
Abnormal Carotid-2 & $229(125 \sim 230)$ & $2.5(2.0 \sim 4.0)$ & Moderate \\
Viscosity: 3.5 mPa-s & & $3(2.0 \sim 4.0)$ & Moderate \\
Abnormal Carotid-2 & $210(125 \sim 230)$ & $3.2(2.0 \sim 4.0)$ & Moderate \\
Viscosity: $7 \mathrm{mPa}-\mathrm{s}$ & & & \\
Abnormal Carotid-2 & $190(125 \sim 230)$ & & \\
Viscosity: $10 \mathrm{mPa}-\mathrm{s}$ & & &
\end{tabular}

\section{CONCLUSION}

In this study, the viscosity varition effects in different realistic carotid atherosclerosis models have been studied using CFD technique and consequently simulated ultrasound based color flow image have been tested and investigated. The blood flow dynamics have been examined under different atherosclerosis and viscosity conditions. The simulated ultrasound based flow images have been compared with CFD flow images and a good matching has been found between them. It was perceived that noticeable increase of the blood flow velocity in carotid atherosclerotic growths of the abnormal artery while velocity looks like a uniform in the normal carotid artery. The severity stages of stenosis have been determined using ICA and CCA velocity information. Most importantly, the models have been examined with varying viscosity as viscosity elevation is linked with atherosclerosis development. The patterns of blood flow in carotid bifurcated region with atherosclerotic plaque for different levels of viscosities are found more complex in case of elevated viscosity as well as the patterns are different for different viscosities. It has been found that autocorrelation and phase shift based estimated ultrasound color flow velocity imaging can track this viscosity induced contrast. Therefore, an important finding of this study is that viscosity induced contrast is not negligible and significantly, it is detectable by ultrasound scanning. As the conventional ultrasound flow imaging does not consider viscosity changes effect, the result analysis of this study suggests that incorporation of the viscosity changes contrast might improve the ultrasound flow imaging based carotid atherosclerosis diagnosis. We think the findings of this work might be beneficent for better diagnosis of the current conditions of carotid atherosclerosis and predict the forthcoming growths to minimize the risk of brain stroke.

\section{REFERENCES}

[1] W. Johnson, O. Onuma, M. Owolabi, and S. Sachdev, "Stroke: A Global Response is Needed," Bulletin of the World Health Organization, vol. 94, pp. 634-634A, Sep. 2016, doi: 10.2471/BLT.16.181636.

[2] C. Banerjee and M. I. Chimowitz, "Stroke Caused by Atherosclerosis of the Major Intracranial Arteries," Circulation Research, vol. 120, pp. 502-513, 2017, doi: 10.1161/CIRCRESAHA.116.308441.

[3] U. Morbiducci, A. M. Kok, B. R. Kwak, P. H. Stone, D. A. Steinman, and J. J. Wentzel, "Atherosclerosis at arterial bifurcations: evidence for the role of haemodynamics and geometry," Thrombosis and Haemostasis, vol. 115, no. 03, pp. 484-492, 2018.

[4] K. S. Cunningham and A. I. Gotlieb, "The Role of Shear Stress in the Pathogenesis of Atherosclerosis," Laboratory Investigation, vol. 85, pp. 9-23, 2005, doi: 10.1038/labinvest.3700215.

[5] C. Carallo, A. Pujia, C. Irace, M. F. De, C. Motti, and A. Gnasso, "Whole Blood Viscosity and Haematocrit are associated with Internal Carotid Atherosclerosis in Men," Coronary Artery Disease, vol. 9, pp. 113-117, 1998.

[6] E. Ralph, D. O. Holsworth Jr, and J. V. Wright, "Blood Viscosity: The Unifying Parameter in Cardiovascular Disease Risk," Holistic Primary Care, vol. 13, pp. 1-2, 2012.

[7] R. C. Becker, "The Role of Blood Viscosity in the Development and Progression of Coronary Artery Disease," Cleveland Clinic journal of medicine, vol. 60, pp. 353-358, 1993.

[8] E. D. Taco-Vasquez et al., "Association between Blood Viscosity and Cardiovascular Risk Factors in Patients with Arterial Hypertension in a High Altitude Setting," Cureus, vol. 11, no. 1, Jan 2019, Art. no. e3925, doi: 10.7759 /cureus.3925.

[9] C. S. G. Murray, T. Nahar, H. Kalashyan, H. Becher, and N. C. Nanda, "Ultrasound Assessment of Carotid Arteries: Current Concepts, Methodologies, Diagnostic Criteria, and Technological Advancements," Echocardiography, vol. 35, no. 12, pp. 2079-2091, Dec. 2018, doi: 10.1111/echo.14197

[10] A. A. Oglat et al., "A Review of Medical Doppler Ultrasonography of Blood Flow in General and Especially in Common Carotid Artery," Journal of medical ultrasound, vol. 26, no. 1, pp. 3-13, Jan. 2018, doi: 10.4103/JMU.JMU_11_17. 
[11] D. Gaitini and M. Soudack, "Diagnosing Carotid Stenosis by Doppler Sonography: State of the Art," Jorurnal of Ultrasound in Medicine, vol. 24, no. 8, pp. 1127-1136, Aug 2005, doi: 10.7863/jum.2005.24.8.1127.

[12] S. N. Doost, D. Ghista, B. Su, L. Zhong, and Y. S. Morsi, "Heart Blood Flow Simulation: A Perspective Review," Biomedical Engineering Online, vol. 15, Dec 2016, doi: 10.1186/s12938-016-0224-8.

[13] D. A. Steinman, D. A. Vorp, and C. R. Ethier, "Computational Modeling of Arterial Biomechanics: Insights into Pathogenesis and Treatment of Vascular Diseas," Journal of Vascular Surgery, vol. 37, no. 5, pp. 1118-1128, May 2003, doi: 10.1067/mva.2003.122.

[14] A. Swillens, L. Løvstakken, J. Kips, H. Torp, and P.Segers, "Ultrasound Simulation of Complex Flow Velocity Fields based on Computational Fluid Dynamics," IEEE Transactions on Ultrasonics, Ferroelectrics, and Frequency Control, vol. 56 , pp. 546 - 556, March 2009, doi: 10.1109/TUFFC.2009.1071.

[15] E. Y. Wong et al., "Doppler ultrasound and numerical analysis for the assessment of hemodynamic disturbances in ulcerated carotid arteries," 2008 IEEE Ultrasonics Symposium, Beijing, China, 2008, pp. 1603-1606, doi: 10.1109/ULTSYM.2008.0391.

[16] J. Dong, K. Inthavong, and J. Tu, "Image-Based Computational Hemodynamics Evaluation of Atherosclerotic Carotid Bifurcation Models," Computers in Biology and Medicine, vol. 43, no. 10, pp. 1353-1362, Oct. 2013, doi: 10.1016/j.compbiomed.2013.06.013

[17] D. K. Saha and A. B. M. Aowlad Hossain, "A simulation study on viscosity change effects in ultrasound based carotid atherosclerosis diagnosis," 2015 International Conference on Advances in Electrical Engineering (ICAEE), Dhaka, Bangladesh, 2015, pp. 68-71, doi: 10.1109/ICAEE.2015.7506798.

[18] R. M. Nerem, "Vascular Fluid Mechanics, the Arterial Wall, and Atherosclerosis," Journal of Biomechanical Engineering, vol. 114, no. 3, pp. 274-282, 1992, doi: 10.1115/1.2891384.

[19] N. Bessonov, A. Sequeira, S. Simakov, Yu. Vassilevskii, and V. Volpert, "Methods of Blood Flow Modelling," Math. Model. Nat. Phenom., vol. 11, no. 1, pp. 1-25, 2016, doi: 10.1051/mmnp/201611101.

[20] J. Donea and A. Huerta, "Finite Element Methods for Flow Problems," John Wiley \& Sons, 2003.

[21] J. A. Jensen, "Field: A Program for Simulating Ultrasound Systems," Medical \& Biological Engineering \& Computing, vol. 34, pp. 351-353, 1996, doi: 10.1.1.50.4778

[22] J. A. Jensen, "A Model for the Propagation and Scattering of Ultrasound in Tissue," Journal of the Acoustical Society of America, vol. 89, pp. 182-190, 1991, doi: 10.1121/1.400497.

[23] M. Schlaikjer, S. Torp-Pedersen, and J. A. Jensen, "Simulation of RF Data with Tissue Motion for Optimizing Stationary Echo Canceling Filters," Ultrasonics, vol. 41, pp. 415-419, 2003, doi: 10.1016/S0041-624X(03)00150-1.

[24] D. H. Evans and W. N. McDicken, "Doppler Ultrasound: Physics, Instrumentation, and Signal Processing," John Wiley \& Sons, Chichester, UK, 2000.

[25] C. Kasai, K. Namekawa, A. Koyano and R. Omoto, "Real-Time Two-Dimensional Blood Flow Imaging Using an Autocorrelation Technique," in IEEE Transactions on Sonics and Ultrasonics, vol. 32, no. 3, pp. 458-464, May 1985, doi: 10.1109/T-SU.1985.31615.

[26] J. A. Jensen, "Estimation of Blood Velocities using Ultrasound: A Signal Processing Approach," Cambridge University Press, New York, 1996.

[27] W. D. Barber, J. W. Eberhard and S. G. Karr, "A New Time Domain Technique for Velocity Measurements Using Doppler Ultrasound," in IEEE Transactions on Biomedical Engineering, vol. BME-32, no. 3, pp. 213-229, Mar. 1985, doi: 10.1109/TBME.1985.325531.

[28] T. Loupas, J. T. Powers and R. W. Gill, "An axial velocity estimator for ultrasound blood flow imaging, based on a full evaluation of the Doppler equation by means of a two-dimensional autocorrelation approach," in IEEE Transactions on Ultrasonics, Ferroelectrics, and Frequency Control, vol. 42, no. 4, pp. 672-688, Jul. 1995, doi: $10.1109 / 58.393110$.

[29] R. C. Gonzalez and R. E. Woods, "Digital Image Processing," Pearson Education, India, 2009.

[30] E. G. Grant et al., "Carotid Artery Stenosis:Gray-Scale and Doppler US Diagnosis-Society of Radiologists in UltrasoundConsensus Conference," Radiology, vol. 229, no. 2, pp. 340-346, Nov. 2003, doi: 10.1148/radiol.2292030516. 